\title{
Recent Progress in FSMA Microactuator Developments
}

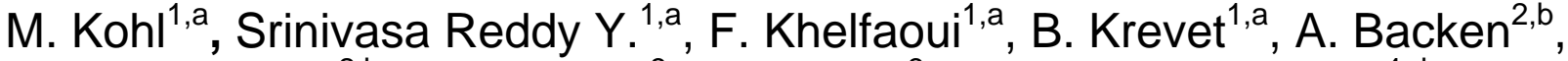 \\ S. Fähler ${ }^{2, b}$, T. Eichhorn ${ }^{3, c}$, G. Jakob ${ }^{3, c}$ and A. Mecklenburg ${ }^{4, d}$ \\ ${ }^{1}$ University of Karlsruhe, IMT, Kaiserstr. 12, 76131 Karlsruhe, Germany \\ ${ }^{2}$ IFW Dresden, P.O. Box 270116, 01171 Dresden, Germany \\ ${ }^{3}$ University of Mainz, Staudinger Weg 7, 55128 Mainz, Germany \\ ${ }^{4}$ Helmholtz-Zentrum Berlin, Glienicker Strasse 100, 14109 Berlin, Germany \\ amanfred.kohı@imt.fzk.de, bs.faehler@ifw-dresden.de, cgerhard.jakob@uni-mainz.de, \\ dmecklenburg@hmi.de
}

Keywords: ferromagnetic shape memory alloys, thin films, microactuators, thermo-mechanical training, finite element simulation

\begin{abstract}
The giant magneto-strain effect is particularly attractive for actuator applications in micro- and nanometer dimensions as it enables contact-less control of large deformations, which can hardly be achieved by other actuation principles in small space. Two different approaches are being pursued to develop ferromagnetic shape memory (FSMA) microactuators based on the magnetically induced reorientation of martensite variants: (1) the fabrication of free-standing epitaxial Ni-Mn-Ga thin film actuators in a bottom-up manner by magnetron sputtering, substrate release and integration technologies and (2) the top-down approach of thickness reduction of bulk Ni-Mn-Ga single crystals to foil specimens of decreasing thicknesses $(200-40 \mu \mathrm{m})$ and subsequent integration. This review describes the fabrication technologies, procedures for thermo-mechanical training adapted to the quasi-two-dimensional geometries of film and foil specimens as well as the performance characteristics of state-of-the art actuators after processing and training.
\end{abstract}

\section{Introduction}

In recent years, ferromagnetic shape memory alloys (FSMAs) have been established as a new class of materials, which show a unique coupling of their mechanical, thermal and ferromagnetic properties causing highly interesting phenomena such as the giant magneto-strain [1] and giant magneto-caloric effect [2]. Fig. 1 shows a schematic of different coupling effects being of interest for actuator applications. Bulk Ni-Mn-Ga single crystals, for instance, reach large magneto strains in the order of $10 \%$ due to magnetically induced reorientation (MIR) of martensite variants connected by easily movable twin boundaries [3-5]. The key requirements for large magneto strains are ferromagnetic martensites in a single variant state, having high magnetocrystalline anisotropy and a low twinning stress [5]. The MIR effect enables contactless control of large deformations at relatively large bandwidths, which are important prerequisites for applications in small space [6]. Recently, also other mechanisms for FSMA microactuation have been explored such as the induction of phase transformation in Ni-Mn-Ga thin films under high magnetic fields (magnetically induced martensite, MIM) [7,8], the antagonism of thermally induced shape recovery force and ferromagnetic attraction force in a magnetic field gradient [9-11], or utilizing a film's magnetic stray field energy to induce a preferential variant orientation [12].

Recent efforts mostly concentrate on the development of FSMA microactuators based on the MIR effect since MIR does not require a phase transformation, which has advantages in terms of power requirements and actuation frequencies. Much work is currently undertaken to fabricate epitaxial FSMA films showing magneto-mechanical properties similar to single crystalline bulk materials [13-16]. Epitaxial films constrained by a rigid substrate, however, do not show useful deformations due to the low blocking stress of a few MPa. In the special case of orthorhombic martensite, MIR has been demonstrated for epitaxial Ni-Mn-Ga film-substrate composites as a redistribution of 
variants is possible without significant change of strain [16]. In order to achieve large deformations, it is essential to develop deposition and substrate-release technologies allowing for free-standing epitaxial FSMA films or free-standing FSMA films, which are highly oriented $[14,17]$.

The following review presents technology routes for fabrication of epitaxial Ni-Mn-Ga film actuators including magnetron sputtering, substrate release and integration technologies. Also the alternative top-down approach of thickness reduction of Ni-Mn-Ga bulk single crystals is described, which inherently gives rise to free-standing single crystalline specimens $[18,19]$. However, the thinning process may considerably affect the microstructure and, thus, variant reorientation and mobility of twin boundaries [18]. The technology impact on magnetization and magneto-strain behavior is discussed and training methods are presented to enable MIR in Ni-Mn-Ga foil actuators.

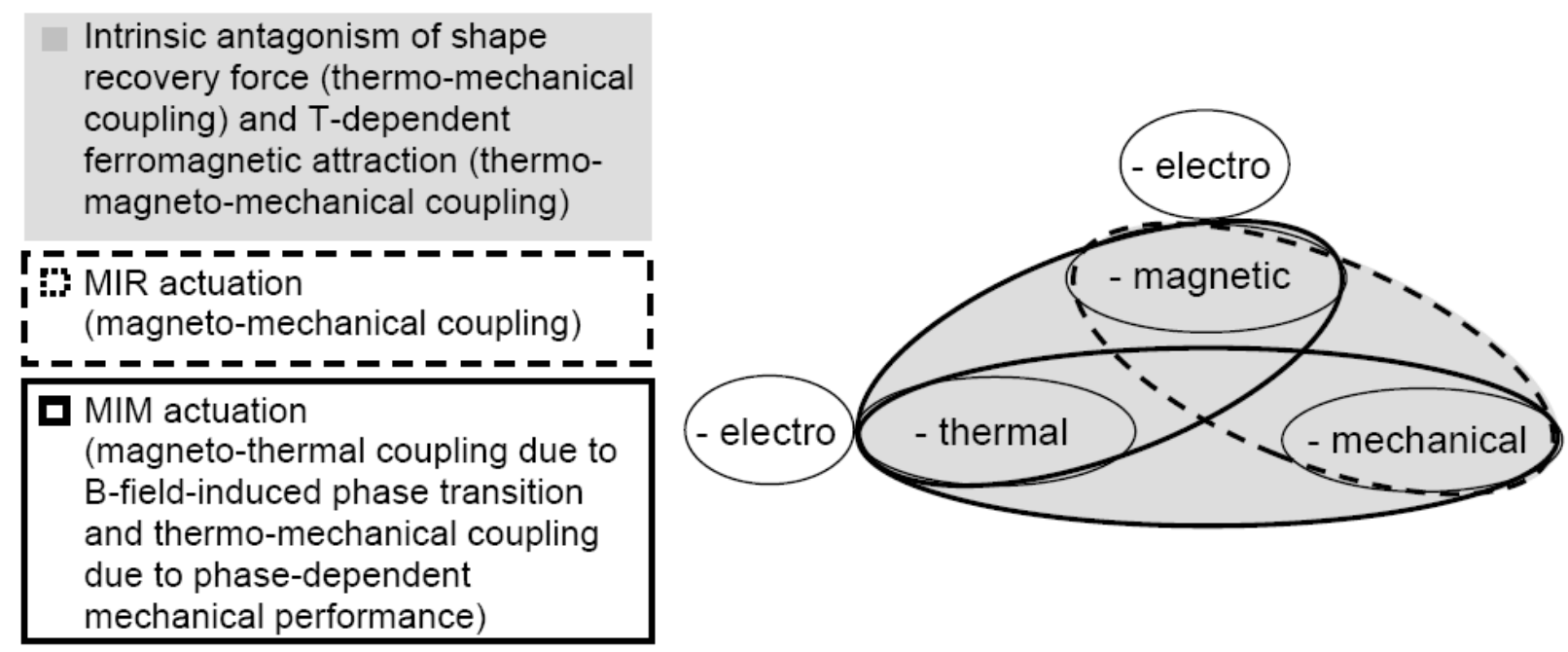

Fig. 1: Schematic of different coupling effects in FSMA materials being of interest for actuator applications. In most cases, it is convenient to use electro-thermal actuation (Joule effect) and electro-dynamic generation of a magnetic field (Ampere's law), which introduces additional coupling effects.

\section{MIR Actuation in Ni-Mn-Ga Thin Films}

Fig. 2a shows a simplified representation of the MIR actuation process in bulk Ni-Mn-Ga single crystals. For both crystallographic structures showing MIR (10M and 14M) the short c-axis coincides with the magnetic easy axis. After transformation from cubic austenite, the martensite crystal consists of a mixture of tetragonal martensite variants having different magnetic and crystallographic orientations. Regions of different c-axis orientation are separated by twin boundaries, which are highly mobile due to the low twinning stress. When the material is exposed to an external magnetic field, the number of martensite variants being in a favorable orientation relative to the field increases at the expense of the other less favorable variants. The maximum possible shape change is given by completely reorienting the crystal from a single variant state with initial c-axis orientation being perpendicular to the magnetic field to a single variant state with final c-axis orientation being parallel to the magnetic field. In this case, the maximum strain is given by the magnitude of tetragonal distorsion $\varepsilon_{\max }=(a-c) / c$.

In bulk Ni-Mn-Ga single crystals, the initial state is usually adjusted by applying a compressive stress perpendicular to the magnetic field as sketched in Fig. 2a. For a thin film specimen, however, this kind of preconditioning is not possible as buckling is likely to occur upon compressive loading in the film plane. In this case, an alternative way of preconditioning has to be developed. Fig. $2 \mathrm{~b}$ shows the option of tensile loading the film along the long side of the specimen, which favors the orientation of one of both a-axis $(10 \mathrm{M})$ being in tensile direction. By applying a magnetic field 


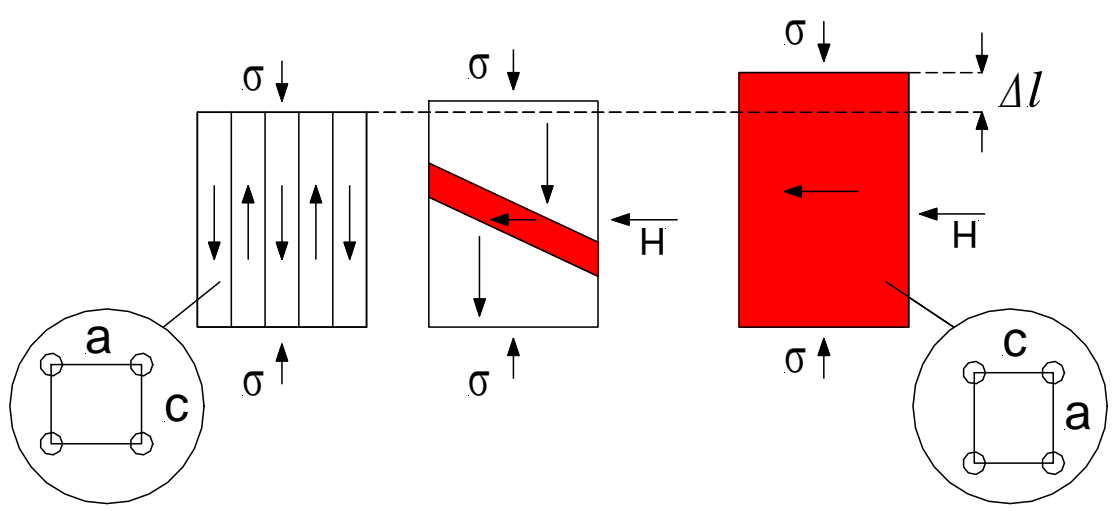

Fig. 2(a): MIR actuation in a bulk Ni-Mn-Ga single crystal for the case of compressive loading.

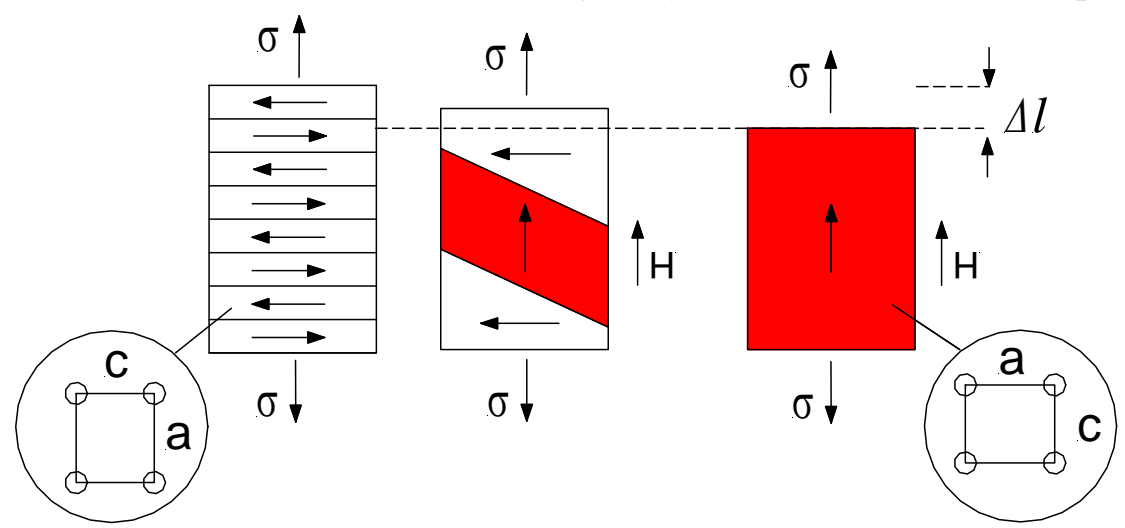

Fig. 2(b): MIR actuation in a single crystalline Ni-Mn-Ga film for the case of tensile loading.

along the tensile direction, a single variant state with final c-axis orientation being parallel to the magnetic field is obtained. In this case, however, the shape change is smaller than $\varepsilon_{\max }$ as this kind of preconditioning does not result in an initial single variant state.

\section{Epitaxial Ni-Mn-Ga Film Actuators}

The development of Ni-Mn-Ga microactuators showing large reorientation strain requires the fabrication of epitaxial or highly oriented Ni-Mn-Ga films, which are at least partially freestanding. In the following, two different technology routes will be described in more detail, the use of a suitable Ni-Mn-Ga film-buffer-substrate combination and the use of a soluble single-crystalline substrate.

Sacrificial layer technology. A typical process flow based on the sacrificial layer technology is shown in Fig. 3. In this case, Ni-Mn-Ga thin films are grown on a $\mathrm{MgO}$ (100) substrate by DC magnetron sputtering covered by a buffer layer. Typical Ni-Mn-Ga film thicknesses range from 0.5 to $2.5 \mu \mathrm{m}$, which are adjusted by the sputtering time. Details on the sputtering process can be found e.g. in Ref. [16]. The distance between the target and substrate is $90 \mathrm{~mm}$, the pressure is $8 \cdot 10^{-3} \mathrm{mbar}$ and the sputtering power is $100 \mathrm{~W}$. The sputtering target is prepared by casting. Since martensitic transitions strongly depend on composition, composition adjustment is a challenge since commonly no stoichiometric transfer from the target to the substrate occurs. The substrate temperature is identified as an essential process parameter. Here we observe an increased loss of Mn and Ga with increasing temperature [20]. Moreover, details of the specific set-up used are crucial, e. g., changing the magnetron configuration by inserting a $\mathrm{Cu}$ plate between target and magnetron results in a composition shift sufficient to switch between NM and 14M martensite [21]. As a consequence, we conclude that adaptation of target composition is required for each set-up individually. The only general guideline we can suggest is to use surplus Mn when using heated substrates, required for epitaxial growth. Indeed, fine tuning of film composition can be done by varying substrate 
temperature. This may be the reason why we observe an optimum temperature range around 350$400{ }^{\circ} \mathrm{C}$ in contrast to other groups.

Epitaxial growth is proven by X-ray diffraction (XRD) $[16,17]$. The commonly used diffraction in Bragg Brentano geometry, however, can be misleading when examining (100) oriented epitaxial films. When the film exhibits the intended martensitic structure at room temperature, the tilt associated with a martensitic distortion results that almost no martensitic variant contributes to the diffraction pattern [22]. Hence, a film exhibiting the intended epitaxy and martensitic state at room temperature appears to have a poor reflection pattern in standard $\theta-2 \theta$ geometry. Clear phase identification is only possible in tilted geometry [22].

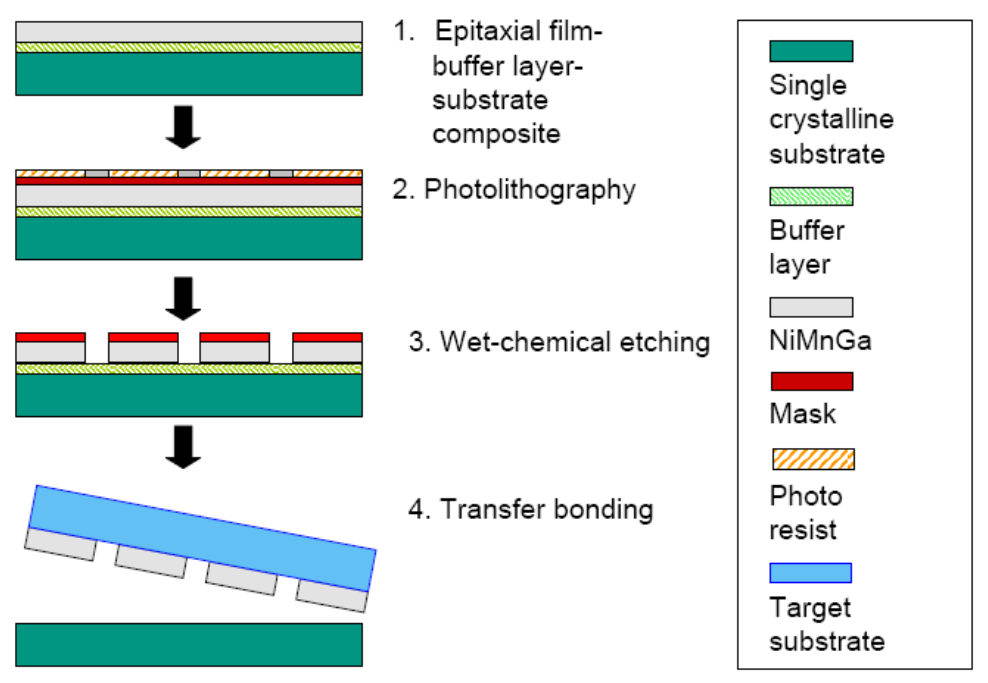

Fig. 3: Process flow for fabrication of epitaxial film actuators based on the sacrificial layer technology.

Micromachining is performed by conventional photolithography and wet chemical etching. In this process step, the $\mathrm{MgO}$ (100) substrate and the buffer layer have to fulfill the basic requirements of low roughness and high chemical stability during the etching process. The buffer layer has, in addition, to provide sufficient bonding strength during processing and to allow for selective removal. $\mathrm{MgO}$ is not a suitable substrate for microsystems applications. Therefore, the Ni-Mn-Ga structures need to be transferred to another substrate (target substrate) after micromachining. For this purpose, novel transfer bonding technologies may by applied, which have originally been developed for the batch integration of high quality materials like single crystalline silicon or shape memory alloys in microsystems [23]. The micromachined target substrate consists of a receiver bonding site and openings to allow for the motion of freely movable Ni-Mn-Ga microparts. First, it is bonded to the epitaxial Ni-Mn-Ga film before the sacrificial buffer layer is removed. Depending on the level of integration, the target substrate also contains electrical interconnections and magnetic structures for the generation of a magnetic field.

Fig. 4(a) shows an epitaxial Ni-Mn-Ga microactuator, which has been transfer bonded to a lasermicromachined alumina substrate for testing purposes. In this case, the microactuator is designed as a microbridge allowing the generation of work perpendicular to the plane of substrate. First performance tests have been made for epitaxial Ni-Mn-Ga bridge microactuators of $2.5 \mu \mathrm{m}$ thickness. The thermoelastic properties are determined in a thermostat, in which the ambient temperature is ramped step-wise in the range between room temperature and $453 \mathrm{~K}$. In between the temperature steps, sufficient waiting time is maintained in order to allow for stationary thermal conditions. The deflection of the center of the freely suspended Ni-Mn-Ga bridge is determined by a video microscope. Fig. 4(b) shows a deflection-temperature characteristic for a constant load of $290 \mu \mathrm{N}$ upon heating and cooling. These results are exemplarily shown for a film pealed off from the $\mathrm{MgO}$ substrate [17]. In the temperature range between 390 and $450 \mathrm{~K}$, a strong change of deflection is observed, which is completely recovered upon thermal cycling. The crystal structure 


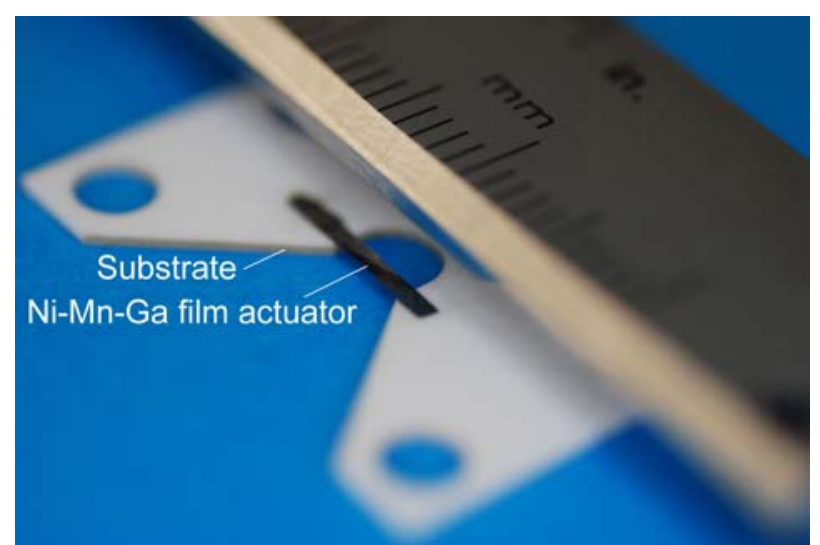

Fig. 4(a): Test actuator of a free-standing epitaxial Ni-Mn-Ga film [17].

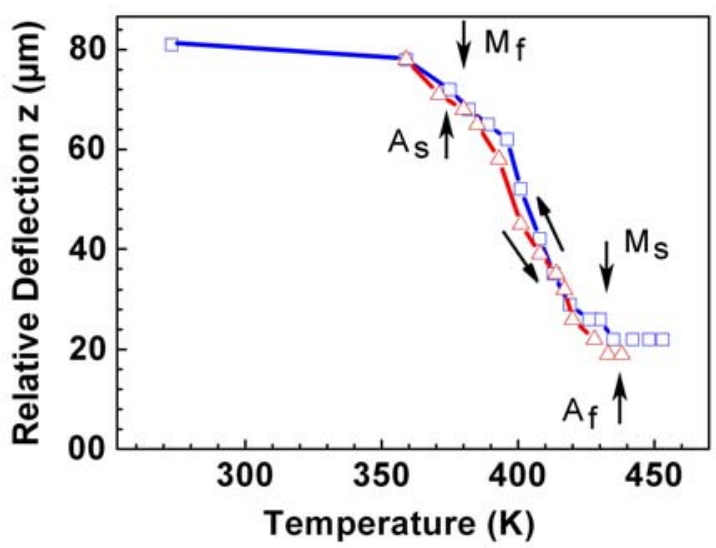

Fig. 4(b): Deflection-temperature characteristic of an epitaxial Ni-Mn-Ga film actuator for a load of $290 \mu \mathrm{N}$ [17].

has been identified to be non-modulated (NM) tetragonal martensite at room temperature. The austenitic and martensitic phase transformation temperatures $A_{\mathrm{s} / f}$ and $M_{\mathrm{s} / f}$ are determined to be $416 / 450 \mathrm{~K}$ and $427 / 398 \mathrm{~K}$, respectively. The Curie temperature is $325 \mathrm{~K}$. Due to the NM microstructure of the epitaxial Ni-Mn-Ga microactuator, so far, only the thermal shape memory effect has been demonstrated.

Sacrificial substrate technology. Fig. 5 depicts the process flow, which has been developed based on the sacrificial substrate technology. The Ni-Mn-Ga thin films are grown on a $\mathrm{NaCl}(100)$ substrate by DC magnetron sputtering at $480{ }^{\circ} \mathrm{C}$. The target has been polished from an arc melted disc of the composition Ni-49, Mn-30.5, Ga-20.5 (at.\%). In this case, typical deposition parameters have been 0.05 mbar argon pressure and $100 \mathrm{~mA}$ current at $320 \mathrm{~V}$ voltage, resulting in a deposition rate of $1.3 \mathrm{~nm} / \mathrm{s}$ at a substrate distance of $30 \mathrm{~mm}$. More information on the sputtering system and process can be found in Ref. [15]. The chemical composition of the films is determined by EDX to be $\mathrm{Ni}_{52.8} \mathrm{Mn}_{30.8} \mathrm{Ga}_{16.4}$ (at. \%). The austenitic and martensitic phase transformation temperatures $A_{s / f}$ and $M_{s / f}$ have been determined to be $338 / 399 \mathrm{~K}$ and $379 / 320 \mathrm{~K}$, respectively. The Curie temperature is $375 \mathrm{~K}$. Released films show a strong texture with (110) orientation contrary to expectations. Further studies are in progress to clarify the influence of substrate and other constraints on the film microstructure.

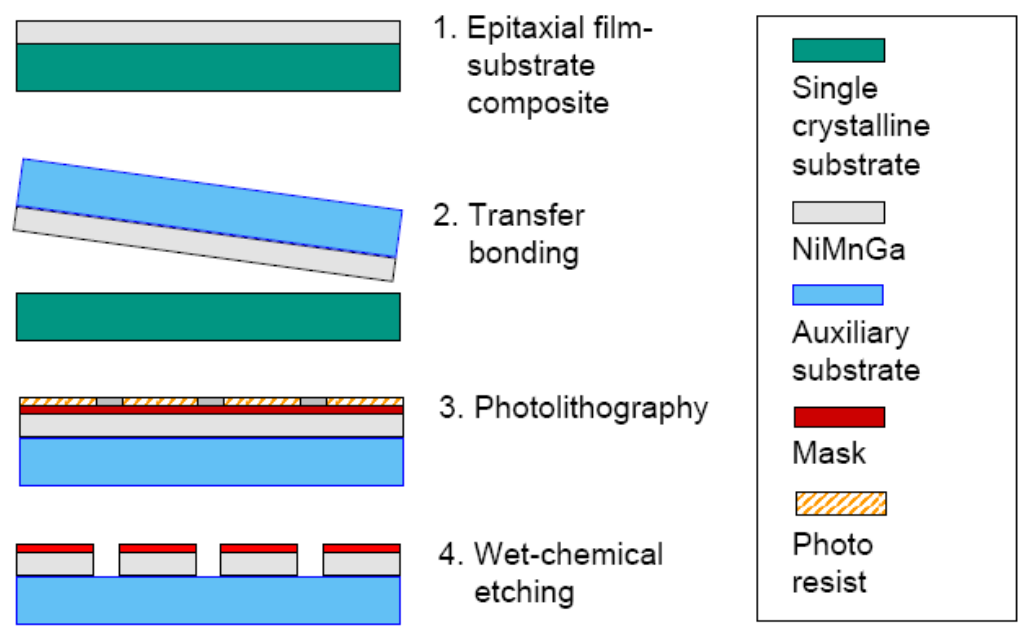

Fig. 5: Process flow for fabrication of epitaxial film actuators based on the sacrificial substrate technology.

After deposition, an auxiliary substrate for the micromachining is bonded to the Ni-Mn-Ga film. Due to the relatively low adhesion between Ni-Mn-Ga film and $\mathrm{NaCl}$ substrate, the composite of $\mathrm{Ni}-\mathrm{Mn}-\mathrm{Ga}$ film and auxiliary substrate can be detached without the need to dissolve the $\mathrm{NaCl}$ 
substrate. Subsequently, micromachining is performed by photolithography and wet chemical etching. Fig. 6(a) shows a corresponding Ni-Mn-Ga test microactuator on a micromachined alumina substrate. A typical deflection-temperature characteristic of a Ni-Mn-Ga film microactuator of $1 \mu \mathrm{m}$ thickness is shown in Fig. 6(b) for a constant load of $1 \mathrm{mN}$ upon heating and cooling. In this case, a reversible thermally induced phase transformation occurs between 320 and $400 \mathrm{~K}$.

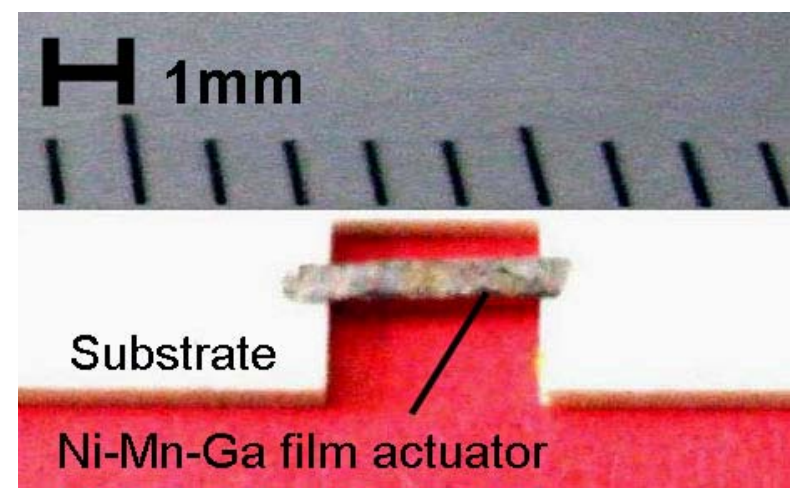

Fig. 6(a): Test actuator of a free-standing NiMn-Ga film actuator of $1 \mu \mathrm{m}$ thickness fabricated by sacrificial substrate technology.

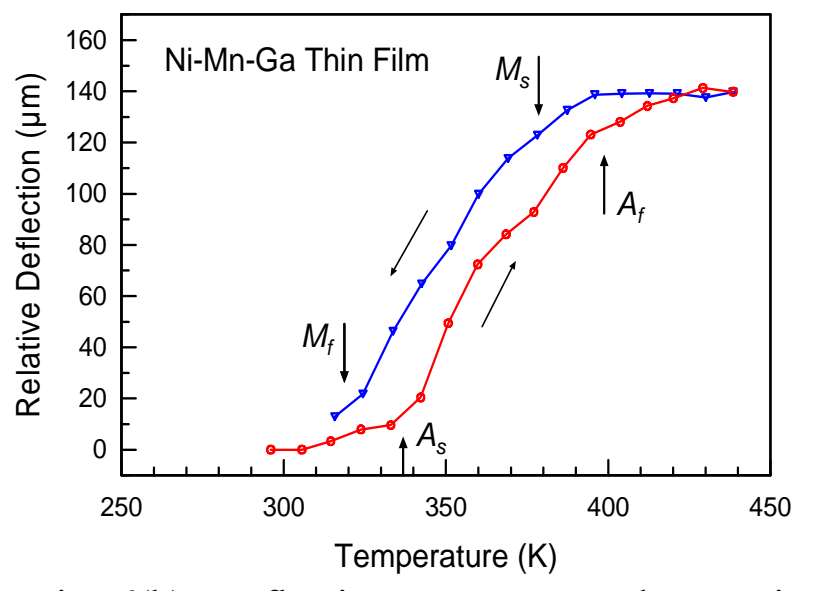

Fig. 6(b): Deflection-temperature characteristic of the Ni-Mn-Ga film actuator shown in Fig. 6(a) for a load of $1 \mathrm{mN}$.

\section{Single-Crystalline Ni-Mn-Ga Foil Actuators}

$\mathrm{Ni}-\mathrm{Mn}-\mathrm{Ga}$ foil actuators have been fabricated by starting with a Ni-Mn-Ga bulk single crystal and subsequent thickness reduction [18]. Ingots of Ni-Mn-Ga have been grown using a SLAg Refinement and Encapsulation (SLARE) technique [24]. The chemical composition has been adjusted to exhibit 5M martensite at room temperature. In a first step, thin plates of $1 \mathrm{~mm}$ thickness are cut from the bulk crystal along the (100) planes by electrical discharge machining. Further steps of thickness reduction include electropolishing, wet-mechanical abrading and mechanical polishing. By this approach, foils of various thicknesses have been produced ranging from 200 down to 60 $\mu \mathrm{m}$. The lateral dimensions of final specimens are about $9 \times 3.2 \mathrm{~mm}^{2}$ and $5.2 \times 3.2 \mathrm{~mm}^{2}$ for the 200 and $60 \mu \mathrm{m}$ foils, respectively.

The chemical composition of the foils are determined by Energy Dispersive X-Ray (EDX) analysis to be about $\mathrm{Ni}_{50.60} \mathrm{Mn}_{28.33} \mathrm{Ga}_{21.06}$ (at.\%). The peak temperatures of martensitic and reverse transformation are determined by differential scanning calorimetry (DSC) to be 327 and $335 \mathrm{~K}$, respectively [18]. The ferromagnetic transition is observed in DSC as a kink at $370 \mathrm{~K}$. The X-ray diffraction pattern of $200 \mu \mathrm{m}$ thick foils determined by $\mathrm{Cu}-\mathrm{K}_{\alpha}$ radiation shows a strong and narrow (400) diffraction peak, which reflects the 5M martensite structure at room temperature similar to the initial bulk single crystal.

The process flow used to micromachine and integrate the Ni-Mn-Ga foil specimens in a microsystem is shown in Fig. 7. In a first step, the Ni-Mn-Ga foil is bonded in vacuum at an elevated temperature on an auxiliary glass substrate using an intermediate layer of a sacrificial polyimide. After micromachining the Ni-Mn-Ga foil by lithography and wet chemical etching, selected micromachined parts are transferred to the target substrate. This is achieved by using a laser-micromachined heat-activated bonding foil on the surface of the target substrate. The bonded microparts are then released from the auxiliary glass substrate by laser ablating the polyimide layer through the glass wafer.

Fig. 8(a) shows a typical stress versus strain characteristic of a single crystalline Ni-Mn-Ga foil of $200 \mu \mathrm{m}$ thickness subjected to tensile loading at room temperature after thickness reduction. A relatively large twinning stress $\sigma_{\mathrm{tw}}$ of $5.5 \mathrm{MPa}$ is observed. The corresponding Ni-Mn-Ga bulk reference actuators, on the other hand, exhibit a much lower twinning stress of about $1.1 \mathrm{MPa}$ in 
compressive mode at room temperature, see Fig. 8(b). At higher temperature, the twinning stress further decreases to about $0.3 \mathrm{MPa}$ at $323 \mathrm{~K}$ [25]. Obviously, the preparation of the foils introduces defects, which impede the mobility of twin boundaries. In fact, $\mathrm{Ni}-\mathrm{Mn}-\mathrm{Ga}$ foil actuators in asreceived condition do not show any magneto-strain effect, while the corresponding Ni-Mn-Ga bulk reference actuators display a large magnetostrain of $6 \%$ above a critical magnetic field of about 0.3 Tesla [25]. These results suggest the need for a suitable training method to reduce the twinning stress and to improve the overall mechanical performance.

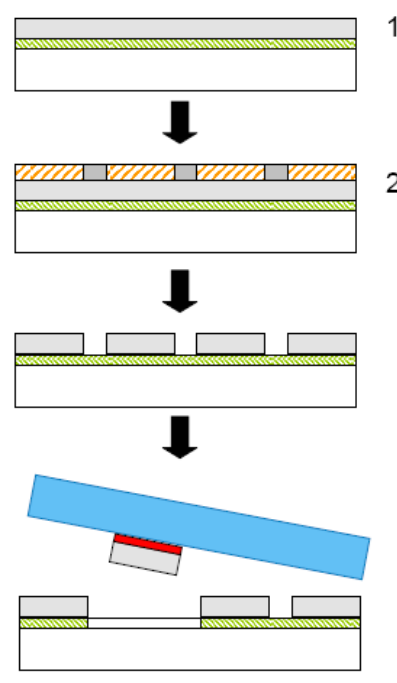

1. Foil integration

2. Photolithography

3. Wet-chemical etching

4. Selective Transfer bonding

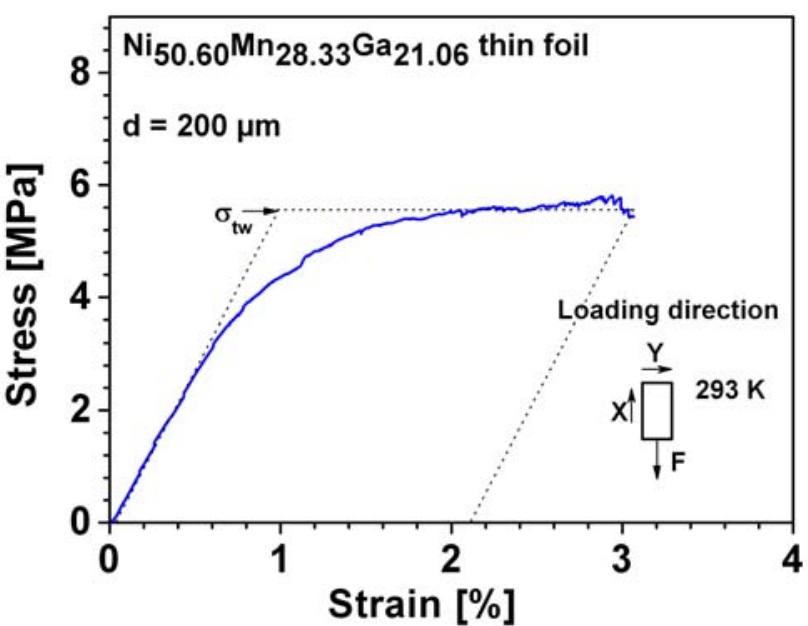

Fig. 8(a): Tensile stress versus strain characteristic of a single crystalline Ni-Mn-Ga foil of $200 \mu \mathrm{m}$ thickness determined at room temperature as-received (a).
Fig. 7: Process flow for micromachining and integration of singlecrystalline Ni-Mn-Ga foil actuators.

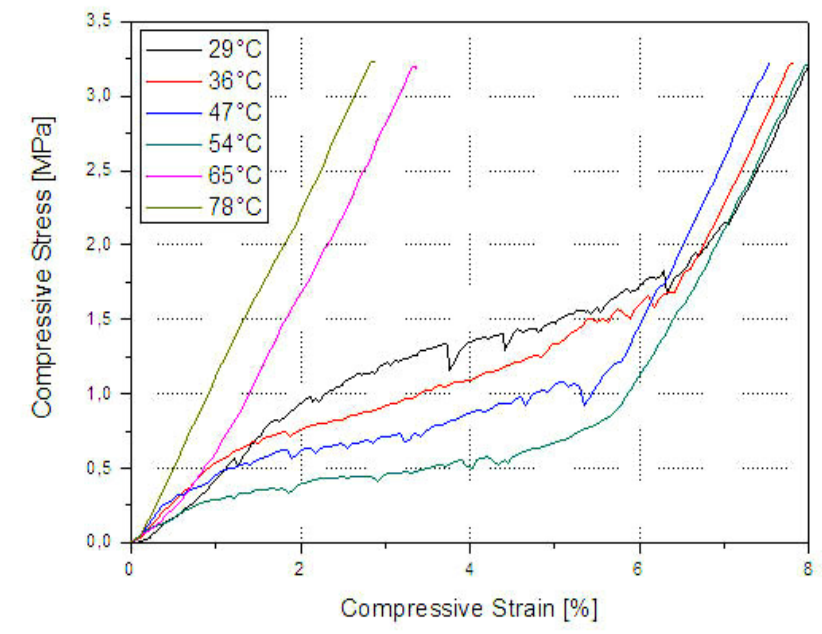

Fig. 8(b): Stress versus strain characteristics of the corresponding bulk single crystalline Ni-Mn$\mathrm{Ga}$ actuator determined at various temperatures as indicated [25].

\section{Thermo-Mechanical Training}

Bulk specimens are usually subjected to initial compressive training in order to improve their mechanical performance. Compression of foils or thin films in lateral direction, however, is not appropriate as buckling effects are likely to occur. Thus, compressive loading may only be applied perpendicular to the foil surface, which appears to be increasingly problematic for decreasing thickness as large contact forces inhibit variant reorientation. Therefore, alternative ways of training the foils have been developed taking into account their quasi-two-dimensional constraints. By thermo-mechanical training in tensile mode, a load is applied along the long side of the foil specimen (X-direction) close to the austenitic finish temperature to induce a quasi-plastic 
deformation at a stress level above the stress plateau. Subsequently, the specimen is thermally cycled several times through the phase transformation regime. This training method allows to preferentially align one of the long a-axis along the tensile direction (X-direction).

The resulting stress versus tensile strain characteristic is shown in Fig. 9 for a single crystalline foil of $200 \mu \mathrm{m}$ thickness at $323 \mathrm{~K}$. As a major result, we find a strong reduction of twinning stress $\sigma_{\mathrm{tw}}$ to about $0.3 \mathrm{MPa}$, which is comparable to the bulk value at $323 \mathrm{~K}$ [25]. We therefore conclude that the tensile training method allows the complete recovery of the low twinning stress of the bulk reference.

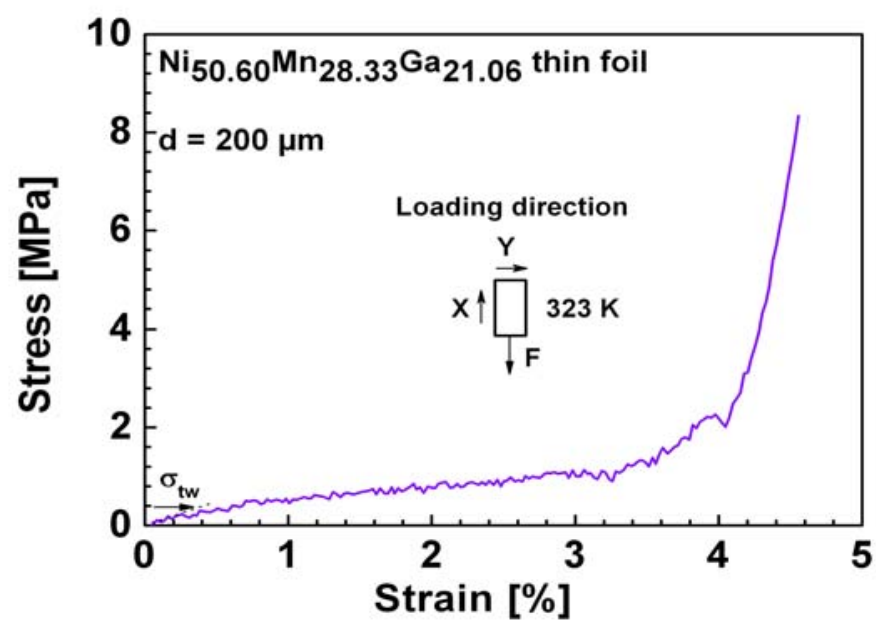

Fig. 9: Tensile stress versus strain characteristic of a single crystalline Ni-Mn-Ga foil of $200 \mu \mathrm{m}$ thickness after thermo-mechanical training in tensile mode.

Magneto-strain experiments are performed in a solenoid setup with maximum homogenous magnetic fields up to about 0.85 Tesla. This setup allows easy optical access to directly observe the magnetic-field-induced deformation of foil actuators by a video microscope. After prealigning the short c-axis by a magnetic field of 0.85 Tesla along the Y-direction of the foil actuator, the deflection of the movable end is monitored by a video microscope while the magnetic field is ramped along the X-direction. Fig. 10 shows a sequence of photos taken during the increase and subsequent decrease of magnetic field for a single crystalline foil actuator of $200 \mu \mathrm{m}$ thickness at room temperature. Relative movements of the setup are eliminated by using a reference marker attached to the substrate. Below the critical magnetic field, the magneto strain keeps almost constant. Upon further increase of the magnetic field, the sample suddenly contracts by about 35 $\mu \mathrm{m}$ in X-direction and then remains deformed until the maximum field of 0.85 Tesla is reached. Due to experimental reasons, the magnetic field is changed only in discrete steps, which limits the accuracy of the critical magnetic field. Upon decreasing the magnetic field, no change of deformation is observed. The magneto-strain characteristics of foil actuators of 60 and $200 \mu \mathrm{m}$ thickness are summarized in Fig. 11. For the $60 \mu \mathrm{m}$ thick foil actuator, a maximum magneto-strain of $1 \%$ is observed. In this case, however, the strain is partially reset upon decreasing magnetic field, which indicates elastic deformation effects associated with reorientation.

Partial reorientation is also observed in magnetization experiments [18]. One reason for the partial reorientation effect is that tensile training only acts on one of the two long a-axis and thus only allows partial prealignment of the martensite variants. Consequently, only half of all martensite variants can reorient. This kind of prealignment favors a unique, parallel alignment of twin boundaries. Hence, intersections of the twin boundaries may act as pinning centers, hindering the twin boundary mobility. Furthermore, pinning of twin boundaries at internal defects cannot be excluded. Depending on the deformation energy required to overcome the pinning of the twin boundaries, reorientation may not be finished before sample magnetization saturates. Additional application of a biasing stress may help to increase the fraction of reorienting martensite variants, 
which remains to be tested in further experiments. An even better prealignment of martensite variants is expected to be achieved by thermo-magneto-mechanical training. In particular, an additional magnetic field applied perpendicular to the tensile loading direction is expected to favor an initial single variant state.
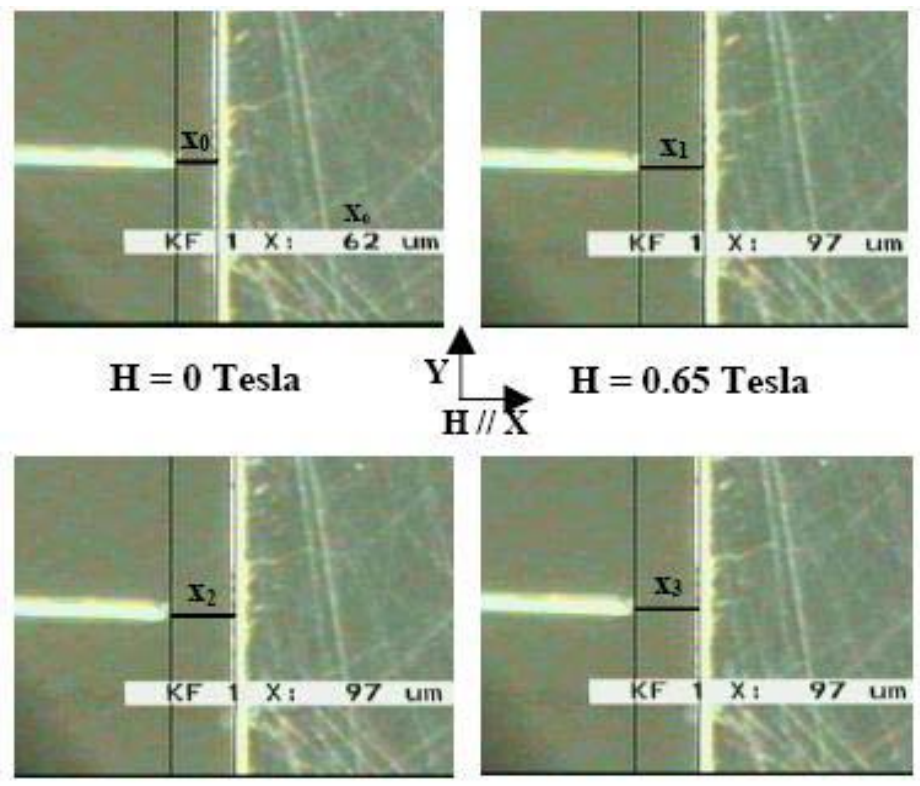

$\mathbf{H}=0.85$ Tesla

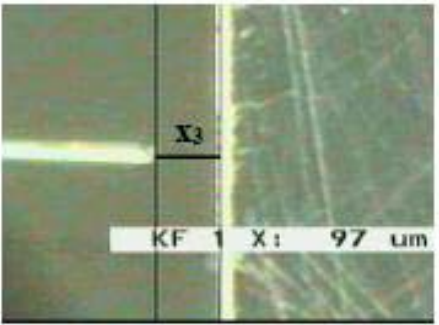

$\mathbf{H}=\mathbf{0}$ Tesla

Fig. 10: Photos of the front end of a single crystalline Ni-Mn-Ga foil actuator of $200 \mu \mathrm{m}$ thickness taken by a video microscope showing magnetic fieldinduced deformation.

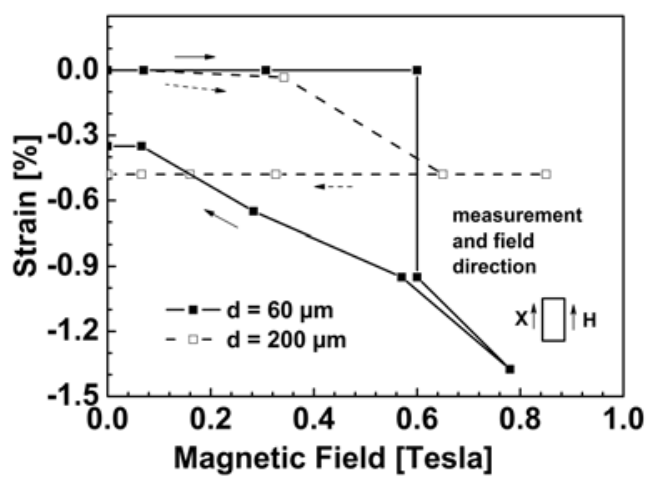

Fig. 11: Magneto-strain characteristics of single crystalline Ni-Mn-Ga foil actuators of 200 and $60 \mu \mathrm{m}$ thickness.

\section{Summary}

FSMA film and foil actuators are currently being developed in order to make use of the MIR effect in small scale applications. Two different technology approaches are described, first, the sacrificial layer as well as the sacrificial substrate technology including FSMA film deposition, substrate release and transfer bonding and, second, the technology of thickness reduction of FSMA bulk single crystals to obtain FSMA foil actuators. As the film and foil actuators cannot be operated by a compressive load in-plane, it is proposed to operate the actuators by a tensile load. For Ni-Mn-Ga film and foil actuators, the MIR effect is induced by applying the magnetic field along the tensile loading direction.

So far, the free-standing film actuators still do not show the right (micro-)structure to observe bulklike magneto-mechanical behavior. In the presented cases, only the thermally induced reorientation of martensite has been demonstrated. Single crystalline Ni-Mn-Ga foil actuators show an initial large twinning stress and thus no measurable magneto strain due to the impact of thickness reduction. It is shown, however, that the low twinning stress of the bulk reference actuators is recovered after thermo-mechanical training in tensile mode. In this case, a partial MIR up to $1 \%$ is directly observed in magneto-strain experiments at zero bias stress. Further improvements on film and foil processing as well as on the training method will be required to achieve bulk-like actuation performance.

\section{References}

[1] For a recent review see, e.g., A.N. Vasil'ev, V.D. Buchel'nikov, T. Takagi, V.V. Khovailo and E.I. Estrin: Physics-Uspekhi 46 (6) (2003) 559-588.

[2] L. Pareti, M. Solzi, F. Albertini and A. Paoluzi: Eur. Phys. J. B 32 (2003) 303-307. 
[3] J. Murray, M. Marioni, S. M. Allen, and R. C. O’Handley: Appl. Phys. Lett. 77 (2000) 886888.

[4] A. Sozinov, A. Likhachev, N. Lanskan, and K. Ullakko: Phys. Lett. 80 (2002) 1746-1748.

[5] O. Heczko, A. Sozinov, and K. Ullakko: IEEE Transactions on Magnetics 36 (5) (2000) 32663268.

[6] M. Kohl, B. Krevet, M. Ohtsuka, D. Brugger and Y. Liu: Materials Transactions 47 (3) (2006) 639-644.

[7] M. Ohtsuka, M. Sanada, M. Matsumoto, T. Takagi, K. Itagaki: Mater. Trans. 44, (2003) p. 2513

[8] M. Ohtsuka, M. Sanada, M. Matsumoto, K. Itagaki: Mater. Sci. Eng. A 378, (2004) p. 377.

[9] M. Kohl, D. Brugger, M. Ohtsuka, T. Takagi: Sensors and Actuator A 114, (2004) p. 445.

[10]D. Brugger, M. Kohl, and B. Krevet: Int. Journal of Appl. Electromagnetics and Mechanics 23 (2006) 99-105.

[11]M. Kohl, D. Brugger, M. Ohtsuka, B. Krevet: Sensors and Actuator A 135, (2007) p. 92.

[12]M. Thomas, O. Heczko, J. Buschbeck, Y. W. Lai, J. McCord, S. Kaufmann, L. Schultz and S. Fähler, Adv. Mat. 21 (2009) p. 1.

[13]J. W. Dong J. W. Dong, L. C. Chen, J. Q. Xie, T. A. R. Müller, D. M. Carr, C. J. Palmstrøm, S. McKernan, Q. Pan, R. D. James: J. Appl. Phys. 88 (2000) 7357-7360.

[14]J. W. Dong, J. Q. Xie, J. Lu, C. Adelmann, C. J. Palmstrøm, J. Cui, Q. Pan, T. W. Shield, R. D. James, S. McKernan: J. Appl. Phys. 95(5) (2004) 2593-2600.

[15]G. Jakob, T. Eichhorn, M. Kallmayer, and H. J. Elmers: Phys. Rev. B76 (2007) p. 174407.

[16]M. Thomas, O. Heczko, J. Buschbeck, U.K. Rößler, J. McCord, N. Scherbaum, L. Schultz and S. Fähler: New J. of Phys. 10 (2008) p. 23040.

[17]F. Khelfaoui, M. Kohl, B. Buschbeck, O. Heczko, S. Fähler, and L. Schultz: Eur. Phys. J. Special Topics, 158, (2008) p. 167.

[18]F. Khelfaoui, M. Kohl, V. Szabo, A. Mecklenburg and R. Schneider, Proc. ICOMAT 08, Santa Fe, USA, (2008), submitted.

[19] O. Heczko, A. Soroka and S.P. Hannula, Appl. Phys. Lett. 93, (2008) p. 022503.

[20] S. Fähler, O. Heczko, M. Thomas, R. Niemann, J. Buschbeck, L. Schultz, Proc. Actuator 2008, 11th Int. Conf. on New Actuators, Bremen, Germany (2008) p. 754.

[21] S. Kaufmann, J. Buschbeck, O. Heczko, L. Schultz, S. Fähler, Proc. ICOMAT 2008, Santa Fe, USA (2008), submitted.

[22] J. Buschbeck, R. Niemann, O. Heczko, M. Thomas, L. Schultz and S. Fähler, Acta Mat. 57 (2009) 2516.

[23]T. Grund, R. Guerre, M. Despont and M. Kohl: Eur. Phys. J. Special Topics 158 (2008) 237242.

[24] A. Mecklenburg, S. Fiechter, H.P. Nabein, R.P. Schneider: Patent WO 2005/099934 A3.

[25]K. Rolfs, A. Mecklenburg, J.-M. Guldbakke, R.C. Wimpory, A. Raatz, J. Hesselbach and R. Schneider, J. Magn. Magn. Mater. (2008), doi:10.1016/j.jmmm.2008.10.023. 